\title{
Remnant-Preserving Anterior Cruciate Ligament Reconstruction Using a Three-Dimensional Fluoroscopic Navigation System
}

\author{
Shuji Taketomi, $\mathrm{MD}^{1}$, Hiroshi Inui, $\mathrm{MD}^{1}$, Takaki Sanada, $\mathrm{MD}^{1}$, Kensuke Nakamura, $\mathrm{MD}^{1}$, \\ Ryota Yamagami, $\mathrm{MD}^{1}$, Hironari Masuda, $\mathrm{MD}^{2}$, Sakae Tanaka, $\mathrm{MD}^{1}$, and Takumi Nakagawa, $\mathrm{MD}^{2}$ \\ ${ }^{1}$ Department of Orthopaedic Surgery, Faculty of Medicine, The University of Tokyo, Tokyo; ${ }^{2}$ Department of Orthopaedic Surgery, Teikyo University School of Medicine, \\ Tokyo, Japan
}

\begin{abstract}
Introduction: Recently, remnant-preserving anterior cruciate ligament (ACL) reconstruction has been increasingly performed to achieve revascularization, cell proliferation, and recovery of high-quality proprioception. However, poor arthroscopic visualization makes accurate socket placement during remnant-preserving ACL reconstruction difficult. This study describes a surgical technique used to create an anatomical femoral socket with a three-dimensional (3D) fluoroscopy based navigation system during technically demanding remnant-preserving ACL reconstruction. Surgical Technique: After a reference frame was attached to the femur, an intraoperative image of the distal femur was obtained, transferred to the navigation system and reconstructed into a 3D image. A navigation computer helped the surgeon visualize the entire lateral wall of the femoral notch and lateral intercondylar ridge, even when the remnant of the ruptured ACL impeded arthroscopic visualization of the bone surface. When a guide was placed, the virtual femoral tunnel overlapped the reconstructed 3D image in real time; therefore, only minimal soft tissue debridement was required.

Materials and Methods: We treated 47 patients with remnant-preserving ACL reconstruction using this system. The center of the femoral socket aperture was calculated according to the quadrant technique using $3 \mathrm{D}$ computed tomography imaging.

Results: The femoral socket locations were considered to be an anatomical footprint in accordance with previous cadaveric studies.

Conclusions: The 3D fluoroscopy-based navigation can assist surgeons in creating anatomical femoral sockets during remnant-preserving ACL reconstruction.
\end{abstract}

Keywords: Anterior cruciate ligament, Remnant, Computer-assisted surgery, Three-dimensional computed tomography

\section{Introduction}

The anterior cruciate ligament (ACL) is a site injured frequently; consequently, ACL reconstructions are widely performed. Despite recent improvements in ACL reconstruction, subsequent

Received January 20, 2014; Revised (1st) March 30, 2014;

(2nd) July 7, 2014; Accepted August 6, 2014

Correspondence to: Shuji Taketomi, MD

Department of Orthopaedic Surgery, Faculty of Medicine,

The University of Tokyo, 7-3-1 Hongo, Bunkyo-ku, Tokyo 113-0033, Japan

Tel: +81-3-3815-5411, Fax: +81-3-3818-4082

E-mail: takeos-tky@umin.ac.jp

This is an Open Access article distributed under the terms of the Creative Commons Attribution Non-Commercial License (http://creativecommons.org/licenses/by-nc/3.0/) which permits unrestricted non-commercial use, distribution, and reproduction in any medium, provided the original work is properly cited. graft ruptures, residual instability, and limited sports performance after surgery remain unresolved issues. To resolve these, preservation of the remnant tissue of ACL has been attracting attention of late. ACL remnants may potentially enhance revascularization and cell proliferation and promote the recovery of high-quality proprioception and stability ${ }^{1-5)}$, and to achieve these, a remnantpreserving ACL reconstruction technique was recently developed with good outcomes ${ }^{6-8)}$. However, poor arthroscopic visualization makes accurate socket placement during remnant-preserving ACL reconstruction difficult.

Since 2007, we have used a three-dimensional (3D) fluoroscopybased navigation system to position the femoral socket accurately and reproducibly during anatomical ACL reconstruction using hamstring tendon grafts or a bone-patellar tendon-bone (BTB) graft $^{9,10)}$. This method is particularly appropriate for remnantpreserving ACL reconstruction because it enables visualization of 

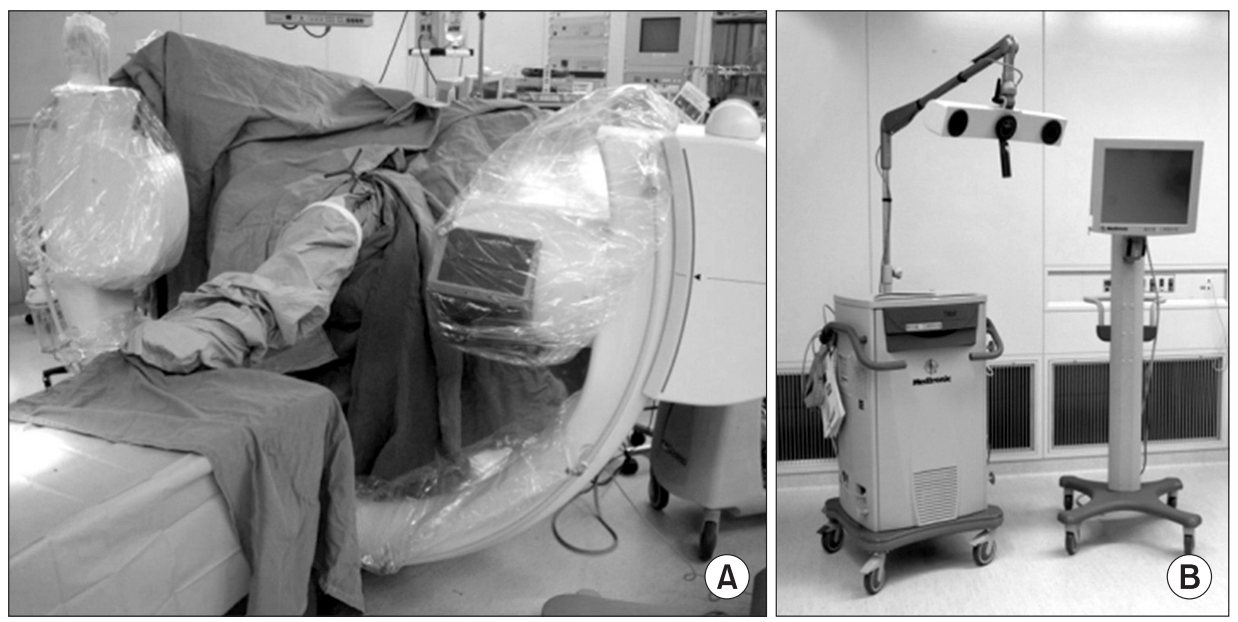

Fig. 1. Image data acquisition and reconstruction. (A) Intraoperative three-dimensional images are acquired with the C-arm. (B) The acquired image data are downloaded to the navigation computer.

the lateral wall and roof of the femoral intercondylar notch, both of which are less easily visualized by arthroscopy. Using this system, the surgeon can identify the lateral intercondylar ridge (LIR), which is an important topographical landmark used to identify the femoral attachment of the ACL with minimal debridement of the remnant ${ }^{11)}$.

The present report aimed to describe remnant-preserving ACL reconstruction using a 3D fluoroscopy-based navigation system and determine the usefulness of this system in the successful creation of an accurately placed femoral socket.

\section{Surgical Technique}

\section{Image Data Acquisition and Reconstruction}

The reference frame (Orthopaedic Frame HC; Medtronic Inc., Louisville, CO, USA) was attached rigidly to the femur with two half-pins at the beginning of surgery. Intraoperative 3D images were acquired with the $\mathrm{C}$-arm of Arcadis Orbic 3D (Siemens AG, Erlangen, Germany) (Fig. 1A). The C-arm of the image intensifier was equipped with a wireless tracker (Stealth Active wireless tracker S/N 130, Medtronic Inc.) to allow image capture and automatic registration by the navigation computer. The acquired image data were downloaded to the navigation computer (StealthStation TRIA plus, Medtronic Inc.) and a 3D image of the distal femur was reconstructed on the computer screen (Fig. 1B). The medial half of the 3D-reconstructed distal femur was deleted using a specific function of the computer software to provide a better view of the lateral wall and the roof of the femoral intercondylar notch (Fig. 2).

\section{Graft Selection, Harvesting, and Preparation}

In most cases, both BTB and hamstring grafts were available.

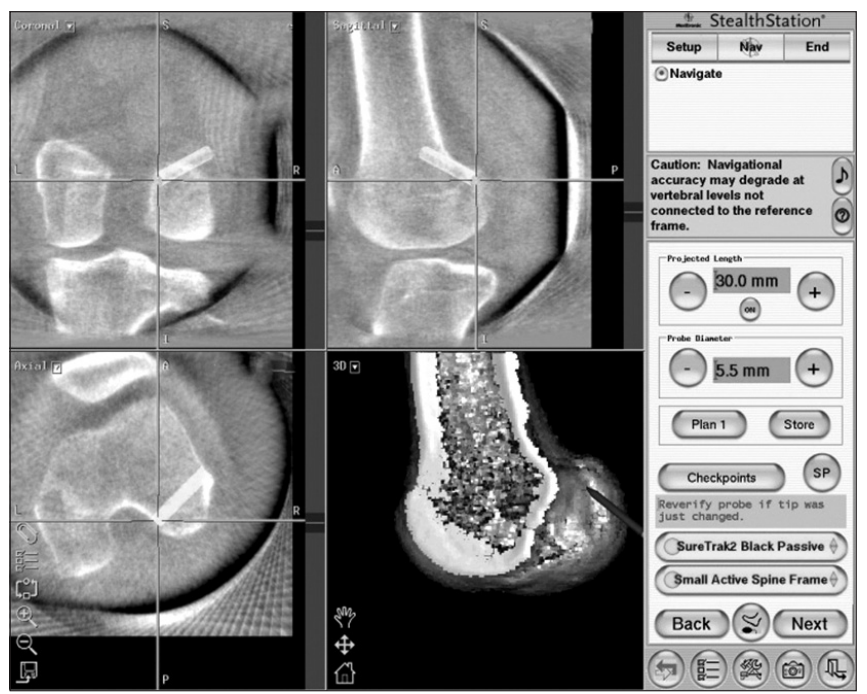

Fig. 2. Navigation views of the right knee. The surface of the lateral wall of the intercondylar notch in an orthogonal projection is visualized on the navigation computer screen. Concurrent axial, coronal, and sagittal two-dimensional images of any point can also be referenced.

Grafts were selected by a surgeon who took into consideration the activity of patients, the types of sports they may be involved in, and patient preference. BTB graft was selected primarily for young male or collision/contact athletes, while hamstring grafts were selected for the others during the study period. BTB grafts were $10 \mathrm{~mm}$ in width and harvested with bone plugs at both ends from the central portion of the patellar tendon. The femoral bone plug for a rectangular socket was usually $5 \times 10 \times 15 \mathrm{~mm}$ as described by Shino et al. ${ }^{12}$. It was connected to an EndoButton (Smith \& Nephew Endoscopy, Andover, MA, USA) using No. 5 and No. 2 FiberWires (Arthrex Inc., Naples, FL, USA). For the hamstring tendon grafts, the semitendinosus tendon was primarily harvested. When the length or diameter of semitendinosus 
tendon was insufficient, the gracilis tendon was also harvested. In most cases, the anteromedial (AM) bundle consisted of a doubled semitendinosus tendon combined with a doubled gracilis tendon, whereas the posterolateral (PL) bundle consisted only of a doubled semitendinosus tendon. The semitendinosus or gracilis tendon may be triple-stranded. The graft for the AM bundle was a minimum of $65-\mathrm{mm}$ in length and 6-mm in diameter, whereas that for the PL bundle was a minimum of $60-\mathrm{mm}$ in length and $5-\mathrm{mm}$ in diameter. The intraosseous graft length should be at least $14 \mathrm{~mm}$. Each graft was connected to an EndoButton CL (Smith \& Nephew Endoscopy) in the usual manner.

\section{Computer Navigation-Assisted Femoral Socket Preparation}

When the remnant tissue of ACL was scarred to the posterior cruciate ligament (PCL) or reattached to either the roof of the notch or the lateral wall, the minimal amount of the residual remnant was cleaned from the lower side to place the guide at the anatomical femoral attachment site using a thermal device.
During debridement of soft tissues, the ACL remnant was retracted medially by a probe. Guide wire placement for femoral socket creation was performed using a femoral guide equipped with a tracker (SureTrak2 Universal Tracker, Large Passive Fighter; Medtronic Inc.). We used a far AM portal technique ${ }^{13)}$ for femoral tunnel creation. With an arthroscope introduced through a lateral or medial portal, the tip of the femoral guide could be placed arthroscopically through a far AM portal at the designated location.

When the remnant tissue impeded guide placement during positioning of the femoral guide through the far AM portal, the remnant tissue of ACL was carefully retracted medially through the medial portal. Image-interactive navigation enabled the surgeon to confirm the position of the tip of the femoral guide on the 3D reconstructed image in real time (Fig. 3). After the tip of the guide was placed at the target point (center of a socket), and after the position of the guide tip was verified on the computer screen, a guide was inserted slightly, the femoral guide tip was
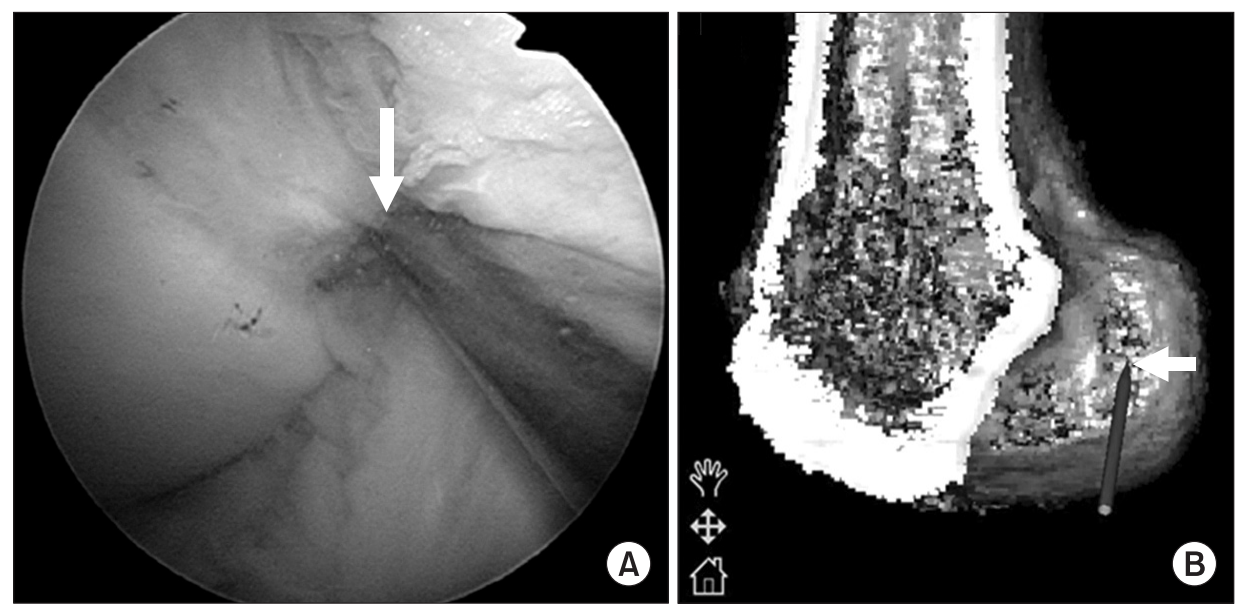

Fig. 3. Arthroscopic and navigation views of the femoral guide. (A) Arthroscopic view of the lateral wall of the femoral intercondylar notch can be obtained through the anteromedial (AM) portal with the knee in $90^{\circ}$ flexion. The tip of the femoral guide is placed within the anatomical femoral footprint through a far AM portal. (B) Navigation view of the lateral wall and roof of the femoral intercondylar notch on the threedimensional reconstructed image. The white arrow shows the tip of the femoral guide.
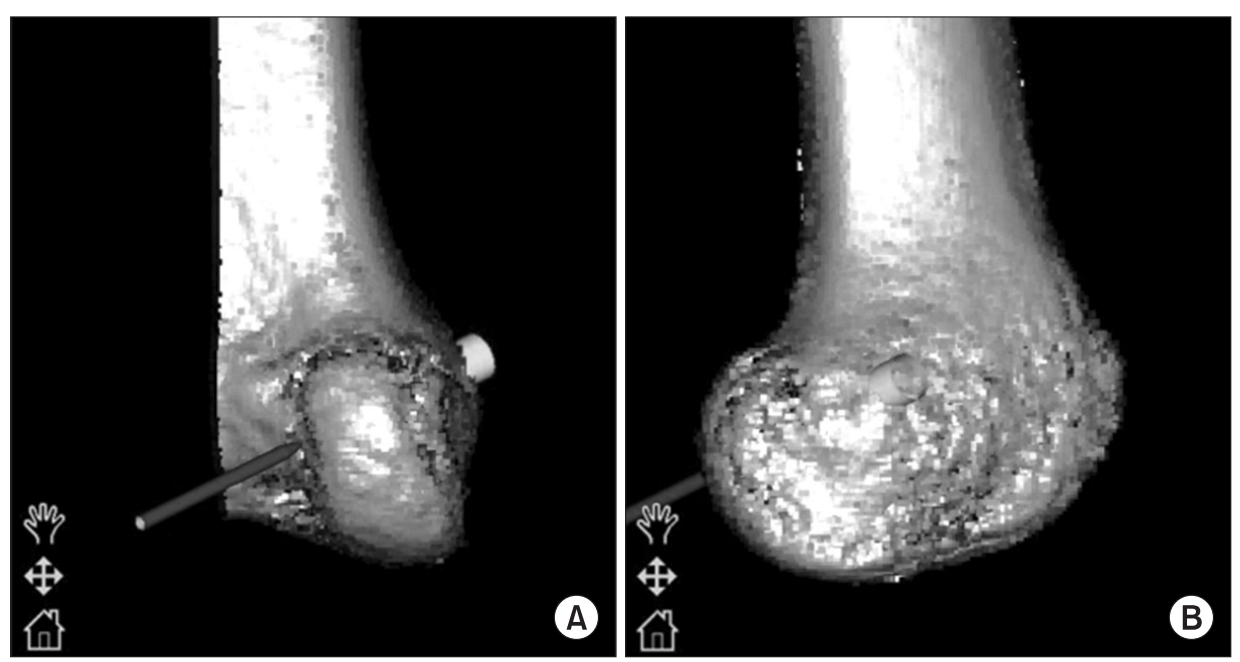

Fig. 4. Navigation view of the right knee. (A) The three-dimensional image is rotated $90^{\circ}$ on the navigation screen in order to evaluate the risk of a back wall blowout. (B) The image is rotated $180^{\circ}$ to show the lateral aspect of the distal femur. 
maintained within the femoral footprint, and the knee was fully flexed. Accordingly, displacement of the guide tip caused by knee flexion could be mostly prevented. On the navigation computer screen, the surgeon could then identify the entire image of the lateral wall of the femoral notch and LIR, even when arthroscopic visualization of the lateral wall of the intercondylar notch was impeded because of the presence of the remnant on deep knee flexion (Fig. 3). If the guide tip was displaced and was not arthroscopically recognized, the surgeon could recognize it on the navigation monitor. Next, the $3 \mathrm{D}$ image was rotated $90^{\circ}$ on the navigation screen where the risk of a back wall blowout could be evaluated (Fig. 4A). Finally, the 3D image was rotated $180^{\circ}$ to reveal the lateral aspect of the distal femur on the navigation screen (Fig. 4B). The total length of the femoral tunnel could thus be evaluated at once. When the BTB graft was used, two parallel guide wires were inserted with a 5-mm distance in the center of the femoral insertion along its long axis (Fig. 5). Conversely, when double-bundle reconstruction with hamstring grafts was performed, two guide wires for the AM and PL sockets were inserted separately at the center of each footprint. After insertion
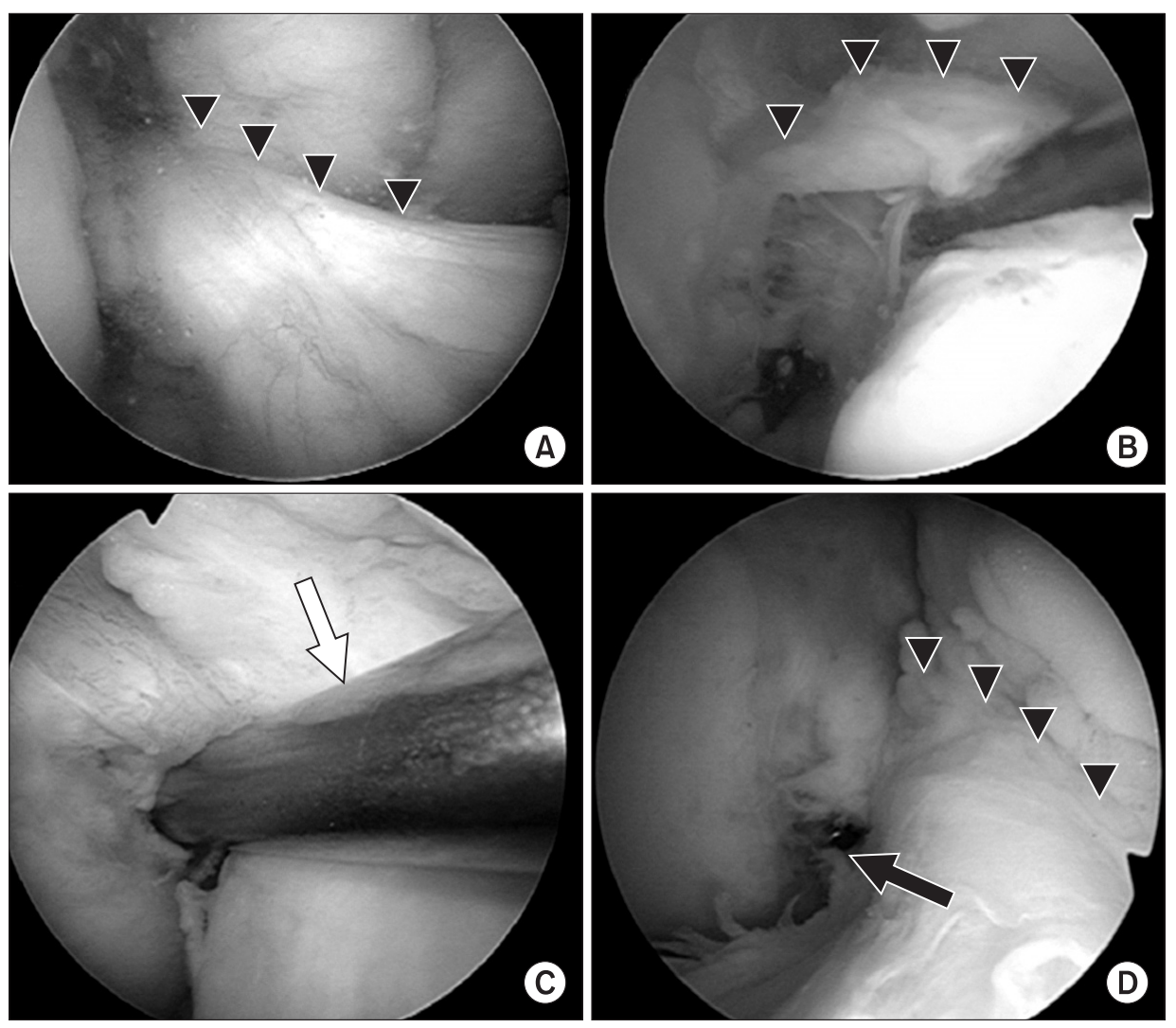

Fig. 5. Intraoperative arthroscopic views of the right knee. (A) The triangles show residual remnant of the torn anterior cruciate ligament (ACL). (B) The ACL remnant is retracted medially by a probe. (C) The femoral guide (white arrow) is placed within the anatomical femoral footprint through a far anteromedial portal. (D) Two parallel guide wires are inserted within the femoral insertion of the ACL. The triangles show the residual remnant of the torn ACL and the black arrow shows the end of the guide pin.
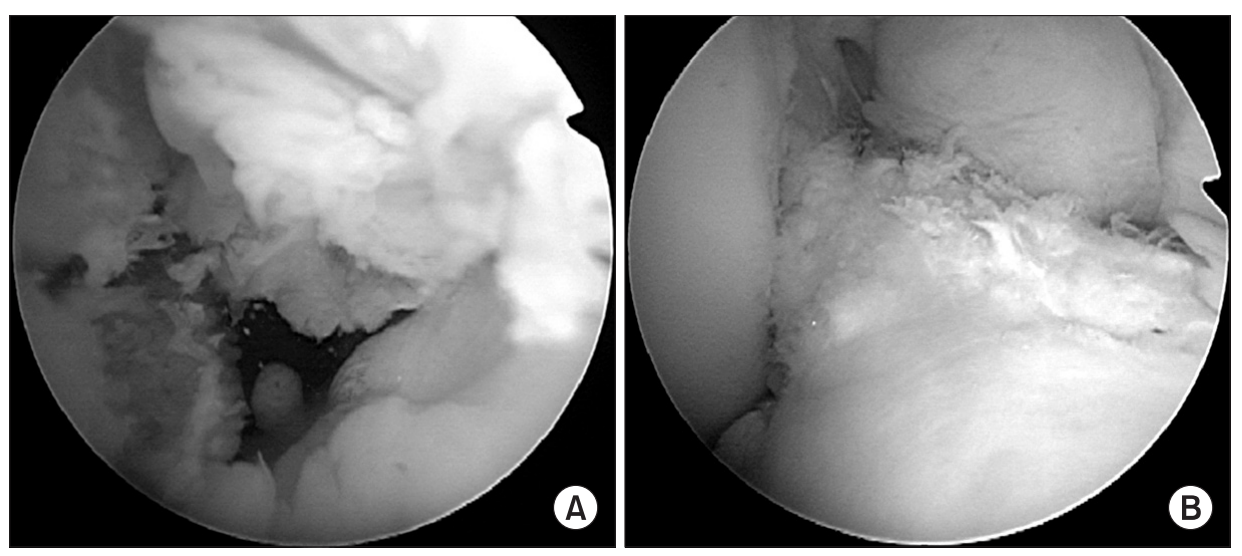

Fig. 6. Intraoperative arthroscopic views of the right knee. (A) Rectangular socket for bone-patellar tendon-bone graft (BTB) grafting. (B) The BTB graft is covered by the preserved remnant of the torn anterior cruciate ligament. 

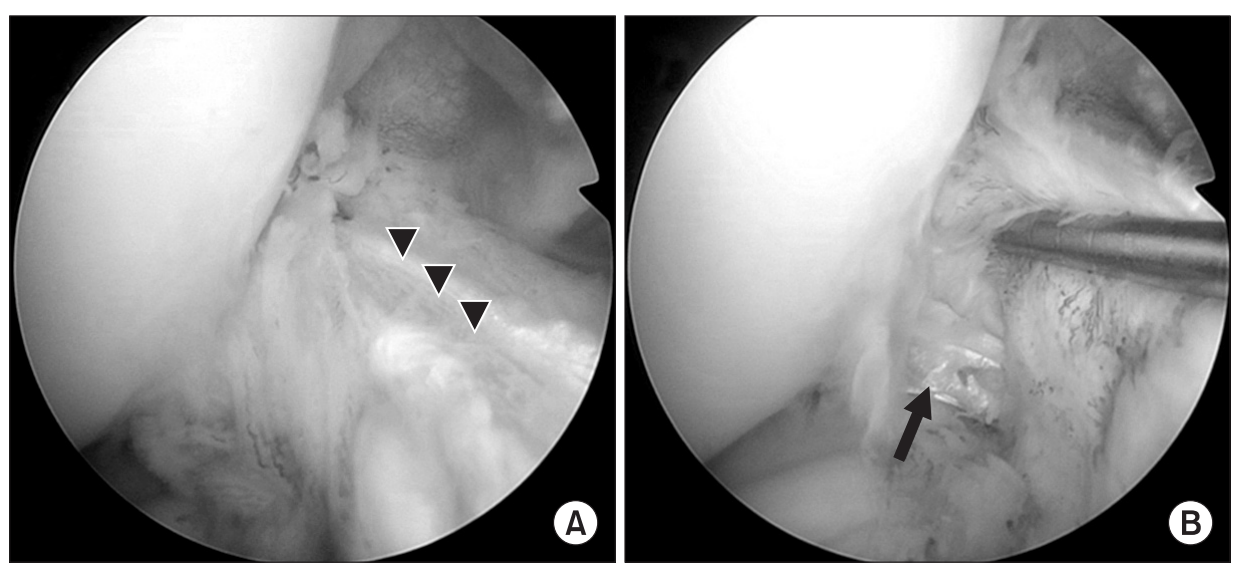

Fig. 7. Intraoperative arthroscopic views of the right knee. Double hamstring grafts are covered by the remnant of the torn anterior cruciate ligament. (A) The triangles show the graft for the anteromedial bundle. (B) The black arrow indicates the graft for the posterolateral bundle. of the guide wires during BTB grafting, they were overdrilled for an appropriate length using a 5-mm cannulated drill, the two tunnels were interconnected using a dilator (Smith \& Nephew Endoscopy), and the lateral femoral cortex was drilled through the center of the two tunnels using an EndoButton drill (Smith \& Nephew Endoscopy) (Fig. 6). When using hamstring tendon(s), each guide wire was overdrilled for an appropriate length by a cannulated drill with a diameter $0.5 \mathrm{~mm}$ smaller than that of the graft, each socket was dilated using a dilator with a diameter similar to that of the graft, and the distant cortex was breached with the EndoButton drill.

The tibial insertion site was arthroscopically determined in reference to the ACL remnant, the medial tibial eminence, the anterior horn of the lateral meniscus, the intermeniscal ligament, and the $\mathrm{PCL}^{14)}$. Tibial tunnel creation and graft passage were performed roughly according to the method described by Ochi et al. ${ }^{15)}$. Briefly, a No. 11 scalpel blade was used to make a longitudinal slit in an ACL remnant tissue. This method allowed not only visualization of the tips of the guide pins or the drill during creation of tibial tunnels but also smooth graft passage. The tibial tunnels were placed in the center of the AM and PL footprints for double-bundle reconstruction. Meanwhile, two parallel guide wires were positioned within the tibial footprint and overdrilled for the entire length using a cannulated drill, and a dilator (Smith \& Nephew Endoscopy) was used to interconnect the two tunnels for rectangular reconstruction using a BTB graft. After creation of the tibial tunnel, a No. 3 or No. 5 polyester braided suture was passed through the eyelet of a passing pin, which was inserted into the femoral tunnel through the longitudinal slit in the remnant tissue of ACL. Subsequently the suture was retrieved by an arthroscopic grasper into the tibial tunnel. The graft was passed through the remnant tissue and the EndoButton loop was flipped outside the femoral cortex in the usual manner. Creation of a longitudinal slit, gradual hooking and pulling up the graft by a probe, and rounding of sharp corners of the bone plug were essential to allow technically challenging graft passage of the BTB through the remnant tissue.

Tibial fixation of the hamstring autografts was accomplished over a suture after fixation using a fully threaded $6.5-\mathrm{mm}$ cancellous screw and washer (Meira Inc., Nagoya, Japan). The AM and PL bundle grafts were fixed at full knee extension and the BTB graft was also fixed at full knee extension. After graft passage, when residual remnant impingement on the roof or the lateral wall of the femoral notch or the PCL was arthroscopically demonstrated, the impinging part of the remnant was shaved off (Fig. 7).

\section{Materials and Methods}

Between January 2011 and December 2013, we treated 47 patients with remnant-preserving ACL reconstruction using the navigation system described above, with the aim of creating a femoral socket with sufficient remnant tissue from the tibia to the femoral notch or the PCL. Eighteen patients underwent remnantpreserving ACL reconstruction using a rectangular BTB graft, while 29 underwent double-bundle reconstruction using hamstring tendon grafts. Remnant-preserving ACL reconstruction was performed in patients with type 1,2, or 3 remnant tissue, as described by Crain et al. ${ }^{16}$. They classified the ACL remnants into 4 morphological patterns: type 1, bridging between the PCL and tibia; type 2, bridging between the roof of the intercondylar notch and tibia; type 3 , bridging between the lateral wall of the intercondylar notch and tibia; and type 4, no substantial ACL remnants. Of the 47 patients ( 22 female and 25 male), 18 had type 1 remnant tissue, 10 had type 2 remnant tissue, and 19 had type 3 remnant tissue. 
There were no patients with partial ACL rupture, including isolated AM or PL bundle rupture. The mean age of the patients was 31 years (range, 16 to 55 years) at the time of surgery. The height and weight [means \pm standard deviations (SDs)] of patients were $166 \pm 9 \mathrm{~cm}$ and $62 \pm 12 \mathrm{~kg}$, respectively. The median duration between injury and surgery was 4 months (range, 1 to 348 months). $3 \mathrm{D}$ computed tomography (CT) imaging of the operated knee was performed 1 week after surgery and the center of the femoral socket aperture was calculated according to the quadrant technique, as described by Bernard et al. ${ }^{17}$. The incidence of a back wall blowout and short femoral tunnel ( $<25 \mathrm{~mm})$ was evaluated. The Institutional Review Board approved this retrospective study. Patients (and their families) were informed that their data would be submitted for publication, and all gave their consent for the same.

\section{Results}

Data in text are given as means \pm standard deviations (range, minimum to maximum). In ACL reconstruction using a rectangular BTB graft, the center of the femoral socket aperture was
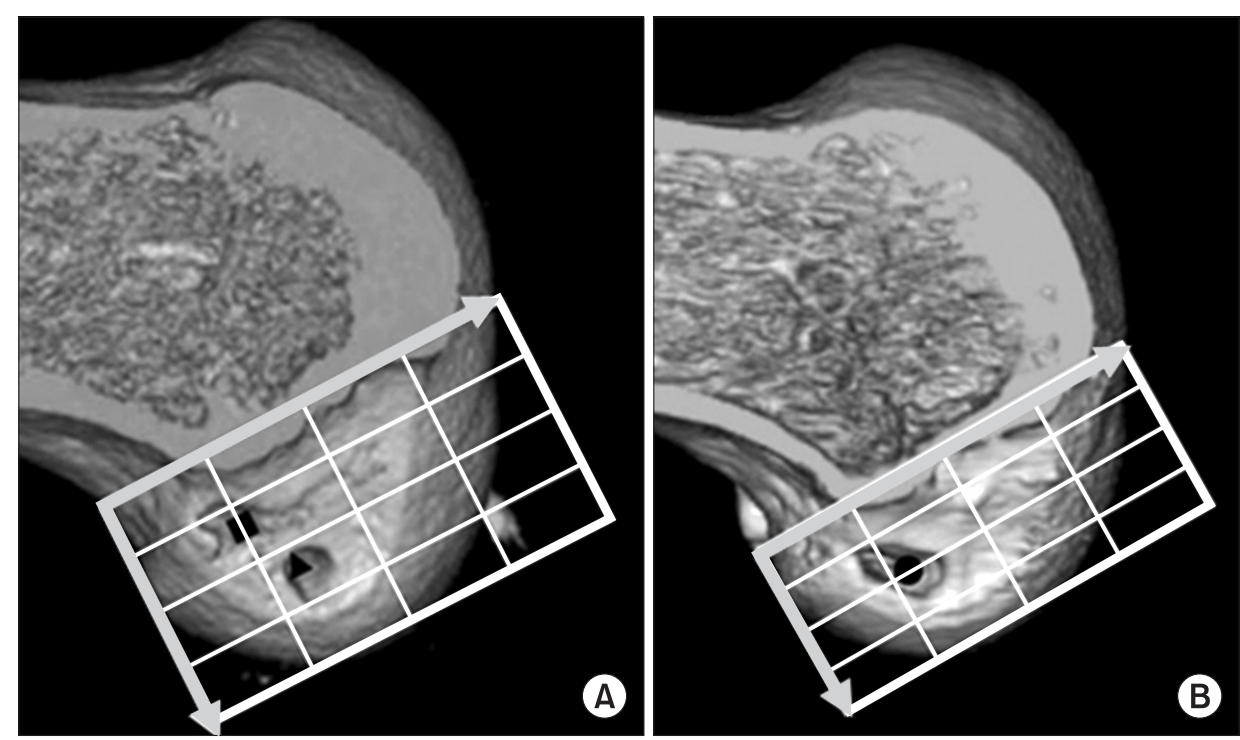

Fig. 8. Femoral socket(s) on three-dimensional computed tomography (3D CT) after surgery. Medial 3D CT view of the reconstructed distal femur at 1 week after surgery shows accurately placed femoral sockets for hamstring tendon graft (A) and an accurately placed rectangular femoral socket for a bone-patellar tendon-bone graft (BTB) (B). Morphometric assessment of femoral tunnel positioning was performed using the quadrant technique, as described by Bernard et al. ${ }^{17)}$. The horizontal position of the femoral tunnel center is defined as the percentage distance from the most posterior contour in reference to the total length of the lateral condyle, whereas its vertical position is defined as the percentage distance from Blumensaat's line in reference to the total lateral intercondylar notch height. For the rectangular tunnel for the BTB grafts, the center of the ellipse by which the rectangular tunnel aperture was approximated was defined as the center of the femoral tunnel for the BTB graft. The black square shows the location of the center of the anteromedial socket, the black triangle shows the location of the posterolateral socket, and the black dot shows the center of the socket location in anterior cruciate ligament reconstruction using a BTB graft.

Table 1. Comparison Between the Cadaver Studies and the Current Study Using the Quadrant Method

\begin{tabular}{|c|c|c|c|c|c|c|}
\hline & \multicolumn{3}{|c|}{ Horizontal position (\%) } & \multicolumn{3}{|c|}{ Vertical position (\%) } \\
\hline & AMB & PLB & Mean & $\mathrm{AMB}$ & PLB & Mean \\
\hline \multicolumn{7}{|l|}{ Current study } \\
\hline Double-bundle & $25.5 \pm 4.7$ & $32.9 \pm 5.6$ & & $34.3 \pm 8.0$ & $57.5 \pm 5.8$ & \\
\hline Bone-patellar tendon-bone & & & $29.1 \pm 4.2$ & & & $51.0 \pm 9.8$ \\
\hline Forsythe et al. ${ }^{18)}$ & 21.7 & 35.1 & 28.4 & 33.2 & 55.3 & 44.3 \\
\hline Lee et al. ${ }^{19)}$ & 34.2 & 38.7 & 36.5 & 26.3 & 53.0 & 39.7 \\
\hline
\end{tabular}

Values are presented as mean \pm standard deviation or number.

AMB: anteromedial bundle, PLB: posterolateral bundle. 
located at $29.1 \% \pm 4.2 \%$ (range, $23.3 \%$ to $41.0 \%$ ) in the horizontal direction and at $51.0 \% \pm 9.8 \%$ (range, $34.5 \%$ to $71.0 \%$ ) in the vertical direction. In double-bundle ACL reconstruction using hamstring tendon grafts, the center of the AM socket aperture was located at $25.5 \% \pm 4.7 \%$ (range, $15.2 \%$ to $33.4 \%$ ) in the horizontal direction and $34.3 \% \pm 8.0 \%$ (range, $19.6 \%$ to $47.2 \%$ ) in the vertical direction, while that of the PL socket aperture was located at $32.9 \% \pm 5.6 \%$ (range, $24.1 \%$ to $43.4 \%$ ) and $57.5 \% \pm 5.8 \%$ (range, $48.5 \%$ to $72.0 \%$ ) in the horizontal and vertical direction, respectively. The femoral socket locations were considered to be anatomical in accordance with previous cadaveric studies examining the positions of the ACL femoral insertion site ${ }^{18,19)}$ (Fig. 8 and Table 1). Neither back wall blowout nor short femoral tunnel was observed.

\section{Discussion}

Computer-assisted surgery has recently been introduced to improve the accuracy and reproducibility of socket placement during ACL reconstruction ${ }^{20-23)}$. We have been using a 3D fluoroscopy-based navigation system for accurate and reproducible placement of the femoral socket through a far AM portal ${ }^{9,10)}$. Using this system, surgeons can identify the LIR, which is an important landmark for ACL femoral insertion not only on arthroscopy but also on $3 \mathrm{D}$ images of the navigation system during primary ACL reconstruction. In remnant-preserving ACL reconstruction, arthroscopic imaging alone is insufficient to evaluate tunnel placement because the residual remnant impedes visualization of the femoral bone surface. Despite poor arthroscopic visualization, this system enables surgeons to visualize the orientation of the lateral wall and the roof of the femoral intercondylar notch. The principle finding of our research was that morphometric analysis of femoral socket placement on postoperative 3D CT images using the quadrant method in this report revealed anatomical femoral socket placements that were similar to cadaveric data previously reported ${ }^{18,19)}$. According to a cadaveric study described by Forsythe et al., the average positions of the center of the AM bundle were $21.7 \%$ in the horizontal direction and $33.2 \%$ in the vertical direction, while those of the PL bundle were $35.1 \%$ and $55.3 \%$ in the respective directions. Calculated from this data, the centers between the AM and PL bundles were located at $28.4 \%$ in the horizontal direction and $44.3 \%$ in the vertical direction. Our data were compared with the cadaveric data using 3D CT images in previous studies (Table 1). Considering these cadaveric data, it was clear that the femoral sockets were anatomically and reproducibly created using $3 \mathrm{D}$ imaging-based navigation in the current series. Furthermore, no complication such as tunnel back wall blowout or short femoral tunnel was observed in the current series.

In our opinion, once the ACL has ruptured, the normal tension cannot be expected in most cases, even though a part of the ACL seems to be intact during arthroscopy. Our concept does not refer to the reconstruction of a partially ruptured ligament with the expectation of maintaining tension in the remaining ligament, rather, it is based on the fact that the remnant tissue only has a supplementary effect and that the aim of remnant-preserving reconstruction is coverage by the conserved ACL tissue. Therefore, the position of the remnant fibers does not affect femoral socket placement using the current technique. Furthermore, decreasing the remnant volume by using the double-bundle reconstruction procedure is acceptable, and the current procedure can be applied to patients in whom the remnant is reattached to the PCL or the roof of the notch.

The current report did not analyze the clinical results of remnant-preserving ACL reconstruction using the navigation system. Further follow-up is required to confirm whether or not this technology will result in good clinical outcomes. It will also be necessary to observe whether or not this procedure is associated with complications such as Cyclops syndrome or pain and/or limited range of knee motion due to impingement on the femoral intercondylar roof, lateral wall, or PCL.

A major problem with the navigation system is the existence of inaccuracy. One of the elements that cause the inaccuracy is a registration error. In the navigation system described in this report, registration based on digital fluoroscopic images which are acquired during surgery is automatically performed; therefore, the registration error can be avoided. According to a laboratory study, 3D fluoroscopic navigation can be performed with an overall application accuracy of $0.47 \pm 0.21 \mathrm{~mm}^{24)}$.

There are other disadvantages associated with the navigation system described in this report. In this system, a reference frame must be fixed to the lateral femur with two half-pins, which necessitates additional skin incisions and drill holes in the femur. Other disadvantages include exposure of the patient and medical staff to radiation at the beginning of the procedure in addition to increased medical costs.

\section{Conclusions}

3D fluoroscopy-based navigation can assist surgeons in creating anatomical femoral sockets during technically challenging remnant-preserving ACL reconstruction. 


\section{Conflict of Interest}

No potential conflict of interest relevant to this article was reported.

\section{References}

1. Schultz RA, Miller DC, Kerr CS, Micheli L. Mechanoreceptors in human cruciate ligaments. A histological study. J Bone Joint Surg Am. 1984;66:1072-6.

2. Georgoulis AD, Pappa L, Moebius U, Malamou-Mitsi V, Pappa S, Papageorgiou CO, Agnantis NJ, Soucacos PN. The presence of proprioceptive mechanoreceptors in the remnants of the ruptured ACL as a possible source of re-innervation of the ACL autograft. Knee Surg Sports Traumatol Arthrosc. 2001;9:364-8.

3. Adachi N, Ochi M, Uchio Y, Iwasa J, Ryoke K, Kuriwaka M. Mechanoreceptors in the anterior cruciate ligament contribute to the joint position sense. Acta Orthop Scand. 2002;73: 330-4.

4. Ochi M, Iwasa J, Uchio Y, Adachi N, Sumen Y. The regeneration of sensory neurones in the reconstruction of the anterior cruciate ligament. J Bone Joint Surg Br. 1999;81:902-6.

5. Ahn JH, Lee SH, Choi SH, Lim TK. Magnetic resonance imaging evaluation of anterior cruciate ligament reconstruction using quadrupled hamstring tendon autografts: comparison of remnant bundle preservation and standard technique. Am J Sports Med. 2010;38:1768-77.

6. Ahn JH, Wang JH, Lee YS, Kim JG, Kang JH, Koh KH. Anterior cruciate ligament reconstruction using remnant preservation and a femoral tensioning technique: clinical and magnetic resonance imaging results. Arthroscopy. 2011;27: 1079-89.

7. Gohil S, Annear PO, Breidahl W. Anterior cruciate ligament reconstruction using autologous double hamstrings: a comparison of standard versus minimal debridement techniques using MRI to assess revascularisation: a randomised prospective study with a one-year follow-up. J Bone Joint Surg Br. 2007;89:1165-71.

8. Yasuda K, Kondo E, Kitamura N, Kawaguchi Y, Kai S, Tanabe Y. A pilot study of anatomic double-bundle anterior cruciate ligament reconstruction with ligament remnant tissue preservation. Arthroscopy. 2012;28:343-53.

9. Nakagawa T, Takeda H, Nakajima K, Nakayama S, Fukai A, Kachi Y, Kawano H, Miura T, Nakamura K. Intraoperative 3-dimensional imaging-based navigation-assisted anatomic double-bundle anterior cruciate ligament reconstruction. Arthroscopy. 2008;24:1161-7.

10. Taketomi S, Nakagawa T, Takeda H, Nakajima K, Nakayama S, Fukai A, Hirota J, Kachi Y, Kawano H, Miura T, Fukui N, Nakamura K. Anatomical placement of double femoral tunnels in anterior cruciate ligament reconstruction: anteromedial tunnel first or posterolateral tunnel first? Knee Surg Sports Traumatol Arthrosc. 2011;19:424-31.

11. Shino K, Suzuki T, Iwahashi T, Mae T, Nakamura N, Nakata $\mathrm{K}$, Nakagawa $\mathrm{S}$. The resident's ridge as an arthroscopic landmark for anatomical femoral tunnel drilling in ACL reconstruction. Knee Surg Sports Traumatol Arthrosc. 2010;18: 1164-8.

12. Shino K, Nakata K, Nakamura N, Toritsuka Y, Horibe S, Nakagawa S, Suzuki T. Rectangular tunnel double-bundle anterior cruciate ligament reconstruction with bone-patellar tendon-bone graft to mimic natural fiber arrangement. Arthroscopy. 2008;24:1178-83.

13. Shino K, Horibe S, Hamada M, Nakamura N, Nakata K, Mae T, Toritsuka Y. Allograft anterior cruciate ligament reconstruction. Tech Knee Surg. 2002;1:78-85.

14. Ferretti M, Doca D, Ingham SM, Cohen M, Fu FH. Bony and soft tissue landmarks of the ACL tibial insertion site: an anatomical study. Knee Surg Sports Traumatol Arthrosc. 2012;20:62-8.

15. Ochi M, Abouheif MM, Kongcharoensombat W, Nakamae A, Adachi N, Deie M. Double bundle arthroscopic Anterior Cruciate Ligament reconstruction with remnant preserving technique using a hamstring autograft. Sports Med Arthrosc Rehabil Ther Technol. 2011;3:30.

16. Crain EH, Fithian DC, Paxton EW, Luetzow WF. Variation in anterior cruciate ligament scar pattern: does the scar pattern affect anterior laxity in anterior cruciate ligamentdeficient knees? Arthroscopy. 2005;21:19-24.

17. Bernard M, Hertel P, Hornung H, Cierpinski T. Femoral insertion of the ACL. Radiographic quadrant method. Am J Knee Surg. 1997;10:14-21.

18. Forsythe B, Kopf S, Wong AK, Martins CA, Anderst W, Tashman S, Fu FH. The location of femoral and tibial tunnels in anatomic double-bundle anterior cruciate ligament reconstruction analyzed by three-dimensional computed tomography models. J Bone Joint Surg Am. 2010;92:1418-26.

19. Lee JK, Lee S, Seong SC, Lee MC. Anatomy of the anterior cruciate ligament insertion sites: comparison of plain radiography and three-dimensional computed tomographic imaging to anatomic dissection. Knee Surg Sports Traumatol 
176 Taketomi et al. 3D Fluoroscopic Navigation in Remnant-Preserving ACL Reconstruction

Arthrosc. 2014 May 10 [Epub]. http://dx.doi.org/10.1007/ s00167-014-3041-2.

20. Colombet PD, Robinson JR. Computer-assisted, anatomic, double-bundle anterior cruciate ligament reconstruction. Arthroscopy. 2008;24:1152-60.

21. Kawakami Y, Hiranaka T, Matsumoto T, Hida Y, Fukui T, Uemoto H, Doita M, Tsuji M, Kurosaka M, Kuroda R. The accuracy of bone tunnel position using fluoroscopic-based navigation system in anterior cruciate ligament reconstruction. Knee Surg Sports Traumatol Arthrosc. 2012;20:150310.

22. Tensho K, Kodaira H, Yasuda G, Yoshimura Y, Narita N,
Morioka S, Kato H, Saito N. Anatomic double-bundle anterior cruciate ligament reconstruction, using CT-based navigation and fiducial markers. Knee Surg Sports Traumatol Arthrosc. 2011;19:378-83.

23. Taketomi S, Inui $H$, Nakamura K, Hirota J, Takei S, Takeda H, Tanaka S, Nakagawa T. Three-dimensional fluoroscopic navigation guidance for femoral tunnel creation in revision anterior cruciate ligament reconstruction. Arthrosc Tech. 2012;1:e95-9.

24. Nolte LP, Beutler T. Basic principles of CAOS. Injury. 2004; 35 Suppl 1:S-A6-16. 